\title{
11. Über die Wirkung einer permanenten mechanischen Ausdehnung auf die optischen Konstanten einiger Metalle; von S. Nakamura.
}

\section{Die Problemstellung.}

Die Wirkung einer mechanischen Deformation auf den Brechungsexponent eines durchsichtigen Körpers ist schon mehrfach untersucht worden. ${ }^{1}$ ) Ein isotroper Körper wird durch die Deformation doppelbrechend, und man hat diese Erscheinung akzidentielle Doppelbrechung genannt. Eine experimentelle Untersuchung über die Änderung der optischen Konstanten der Metalle, welche durch eine mechanische Deformation hervorgerufen wird, fehlt noch. Ein Versuch, welcher mit dem ebengenannten in engem Zusammenhang steht, ist der von Mousson, Tomlinson etc. ${ }^{2}$ ) ausgeführte, über die Änderung des elektrischen Widerstandes eines Drahtes, wenn er gedehnt wird. Hier nimmt der elektrische Widerstand mit dem mechanischen Zug zu; aber wie er sich in der Ebene senkrecht zur Spannung verhält, konnte man natürlich bei einem Draht nicht feststellen.

Die Untersuchung, welche ich hier mitteilen werde, bezieht sich auf die Wirkung einer permanenten mechanischen Ausdehnung auf die optischen Konstanten einiger Metalle. Hr. Prof. Voigt hat mir die Veranlassung gegeben, diesen Versuch durch die Reflexionsmethode zu machen, und er hat mir dazu zwei Polarisationsspektrometer des Göttinger Institutes zur Verfügung gestellt. Die Genauigkeit in meiner Untersuchung ist nicht grob, aber es haben sich doch einige interessante Resultate ergeben, die ich mitteilen möchte. Die genaue $\mathrm{Be}$ stimmung der optischen Konstanten der Metalle ist überhaupt

1) H. Winkelmann, Handb. d. Physik 6. p. 1231.1906.

2) G. Wiedemann, Elekt. u. Magn. 1. p. 520. 
eine schwierige Frage, weil man dazu mit einer Metallfäche völlig frei von Kratzen und Oberflächenschichte arbeiten muB ${ }^{1}$; und wenn es sich um eine Differentialwirkung handelt, ist die Schwierigkeit noch bedeutend größer. Wenn der zu untersuchende Metallspiegel über acht Tage lang auf dem Spektrometer bleiben muß, was gelegentlich bei mir der Fall war, kann man gar nicht behaupten, daB der Oberflächenzustand des Spiegels während dieser Zeit ungeändert geblieben ist. Im Gegenteil, man muß zugeben, daß sich die Oberfläche erheblich geändert hat, selbst wenn, wie bei einigen Versuchen, die Luft in der Nähe des Spiegels mit einer Schale Chlorcalcium getrocknet wird.

Daneben kommt noch die wichtige Frage in Betracht, wie man den zu untersuchenden Spiegel herstellen soll. Wenn man genau wissen will, welcher Art die Wirkung der mechanischen Deformation auf das Metall ist, muB man selbstverständlich zwei Versuche machen; einmal mit einem Spiegel in seinem natürlichen spannungslosen Zustand, und dann mit einem Spiegel aus ausgedehntem Metall. Man kann so verfahren: man schneidet aus einem dicken Metallblech einen Streifen von etwa $30 \mathrm{~cm}$ Länge heraus, glüht ihn in einem Ofen mit Holzkohle aus und läßt ihn sehr langsam abkühlen, so daB er von der anfangs vorhandenen Spannung befreit wird. Nun schneidet man ein Stück von einem Ende des Streifens $a b$ und poliert es. Der übrige Streifen wird jetzt gedehnt, und daraus wird der zweite Spiegel hergestellt. Aber es fragt sich, ob der erste Spiegel wirklich imstande ist, uns die optische Konstante des Metalles in absolut spannungsfreiem $\mathrm{Zu}$ stand zu geben. Worin besteht überhaupt das Polieren. Ich glaube, beim Polieren wie auch beim Feilen, werden die weichen Metallteilchen an der Oberfläche immer wieder durch eine härtere Substanz, das Poliermittel, mitgenommen und lösen sich schlieBlich ab. Wenn beim Polieren die Druckkraft, welche auf den Spiegel ausgeübt wird, groB ist, wird die Oberfläche des Metalles nicht in normalem, sondern in gespanntem Zustande sein. Besonders wenn man einen Polierstabl benutzt und damit über den Spiegel streicht, um eine hochglänzende

1) P. Drude, Wied. Aun. 39. p. 481. 1890. 
Fläche zu erhalten, ist die Oberfläche nicht ganz spannungsfrei, weil ja dieses Verfahren durchaus ähnlich ist dem Ausrollen des Teiges mit einem Rollholz. Man mub deshalb auch befürchten, daß bei der Herstellung des zweiten Spiegels der gewünschte Spannungszustand durch das Polieren sehr viel verschleiert wird. Ich habe daher vorgezogen, statt eines Polierstahles feinen Schmirgel, Zinnasche etc. zu gebrauchen und beim Polieren die Spiegel in allen möglichen Richtungen zu bewegen.

Um möglichst direkt die Wirkung der mechanischen Ausdehnung zu studieren, ist es an sich vielleicht besser, so zu verfahren, wie ich auch beim Silber und Kupfer getan habe. Ich habe da einen Metallstreifen zuerst ausgeglüht, ihn abkühlen lassen, dann in der Mitte poliert, und damit eine Beobachtungsreihe ausgeführt. Dann habe ich den Streifen ausgedehnt und abermals beobachtet. Aber hier stören wieder andere Nachteile; in der ersten Beobachtung für den ungespannten Zustand muB man mit einem langen Streifen arbeiten und kann deshalb nicht einen günstigen Einfallswinkel nehmen, der gewöhnlich etwa $70^{\circ}$ oder $80^{\circ}$ sein soll. Und noch schlimmer ist dje Tatsache, daß die Oberfläche des Spiegels durch den Zug so sehr verschlechtert wird, daB man ohne nochmaliges, allerdings leichteres Polieren den Spiegel kaum benutzen kann.

Für die Ausdehnung der Streifen habe ich die Festigkeitsmaschine im hiesigen technisch-physikalischen Institut benutzt, für deren Gebrauch ich verpflichtet bin, den Herren Prof. Lorentz und Prof. Prandtl meinen aufrichtigen Dank auszusprechen.

\section{Die Beobachtungsmethode.}

Das eine von den zwei Polarispektrometern, welches ich weiter mit (I) bezeichnen werde, ist das seinerzeit von Prof. Drude ${ }^{1}$ ) benutzte. Es war früher mit einem Babin et schen Kompensator versehen, aber dieser ist jetzt weggenommen und statt dessen ist ein Glimmerkompensator angebracht. Die Teilkreise des Polarisators, des Analysators und des Kompensators haben

1) P. Drude, l. c. 
einen gleichen Durchmesser von $8 \mathrm{~cm}$; sie sind in $1 / 2$ Grad geteilt und mit einem Nonius kann man bis zu einer Bogenminute ablesen. Wenn man von der Seite des Ablesefernrohres an die Teilkreise sieht, wächst die Ziffer im Kompensator wie in dem Ubrenzifferblatt, aber bei den beiden Nikolkreisen ist es umgekehrt. Das zweite Spektrometer (II) ist neulich nach Angaben von Prof. Voigt von Fuess angefertigt und von ausgezeichneter Konstruktion. Auch dieses ist mit einem Glimmerkompensator versehen. Die Teilkreise haben einen Durchmesser von $12,5 \mathrm{~cm}$, sind in $1 / 4$ Grade geteilt und haben zwei Nonien, welche bis zu einer Bogenminute ablesen lassen. Die Ziffern in allen drei Kreisen wachsen entgegengesetzt wie die einer Uhr, wenn man sie rom Fernrohr aus sieht. Der einzige Fehler des Apparates ist der, daß die beiden Endflächen des Glan-Thomsonschen Prisma im Polarisator nicht ganz genau parallel sind. Wenn dieser gedreht wird, bewegt sich das Bild des Kollimatorloches in einem kleinen Kreise, dessen Mittelpunkt aber genau auf den Kreuzfaden kommt. Beide Spektrometer sind mit einem gewöhnlichen und auch einem Gaussschen Okular versehen.

Die Nullpunkte des Polarisators und des Analysators sind folgendermaBen bestimmt. Erst wurde der Kompensator weggenommen und das Fernrohr mit dem Analysator dem Kollimator direkt gegenüber eingestellt. Ein Glan-Thomsonsches Prisma wurde auf den Spektrometertisch derart gelegt, daß seine Polarisationsebene beinahe vertikal und seine Endfläche genau senkrecht zur Sehlinie steht (mit dem Gaussschen Okular). Die Polarisationsebene des Polarisators wurde auch beinahe vertikal gemacht. Dann wurde der Analysator in die gekreuzte Lage gebracht und seine Lage abgelesen. Nun wurde das Prisma um $180^{\circ}$ gedreht und die gekreuzte Lage des Analysators wieder abgelesen. Das Mittel der beiden Ablesungen gibt uns den Nullpunkt $a_{0}$ des Analysators. Das Hilfsprisma wurde dann weggenommen und der Nullpunkt $p_{0}$ des Polarisators bestimmt. Unter der Nulllage $c_{0}$ des Kompensators wollen wir diejenige Lage verstehen, wo die Polarisationsebene der langsameren Wellen im Glimmer parallel zur Einfallsebene ist. Diese Lage läßt sich ohne weiteres feststellen, wenn $a_{0}$ und $p_{0}$ bestimmt sind. Im Spektrometer (II) 
war die Fassung für den Kompensator etwas locker, und jedesmal, wenn ich ihn berührt hatte, muBte ich $c_{0}$ erneut bestimmen.

Was die Beleuchtung des Spektrometers anbetrifft, so benutzte ich im Spektrometer(I) einen Linnemannschen Leuchtgas-Sauerstoff brenner mit Natriumkarbonatperlen. Die Flamme war dicht vor dem Kollimatorrohr angebracht, welches nicht mit einem kreisrunden Loch, sondern mit einem Webskischen Spalt versehen war. Die Lichtstärke dieser Lichtquelle erwies sich als nicht genügend, um sehr genaue Einstellungen zu ermöglichen, und daber habe ich mit dem Spektrometer II einen Wülfingschen Monochromator ${ }^{1}$ ) benutzt und mit Sonnenlicht gearbeitet. Von dem Spektrum, welches durch die Monochromatorprismen erzeugt wird, wurde durch einen vertikalen justierbaren Spalt ein sehr schmales Bündel in der Nähe der $D$. Linien abgesondert. Das Lichtbündel ging durch eine Linse von langer Brennweite, welche auf einem horizontalen Spalt des Kollimators ein Bild des vertikalen Monochromatorspaltes abbildete. Hierdurch erhielt ich eine Lichtquelle, die sehr klein und doch hell genug war. Durch die innere Reflexion an verschiedenen Flächen sieht man immer mehrfache Bilder im Gesichtsfelde; das richtige Bild kann man nur dadurch von den anderen trennen, daB man eine kleine Lichtquelle benutzt. Aus demselben Grunde habe ich beim Spektrometer I einen Webskischen Spalt benutzt. Wenn man dort ein rundes Loch klein genug nahm, so war die Lichtstärke so gering, daB man dadurch viel mehr verlor, als man durch die Absonderung des richtigen Bildes gewinnen konnte. Mit einem Webskischen Spalt verbindet man einigermaßen die beiden Vorteile, indem man gerade mit Hilfe des außerhalb des Mittelpunktes vorhandenen Lichtes selbst in der Nähe der kritischen Lage seine Augen sehr leicht auf dem Kreuzfaden haften lassen kann (eine physiologische Frage) und doch für die Feststellung der kritischen Lagen das richtige Bild von anderen Bildern getrennt hat. Bei den Versuchen mit gedehnten Metallplatten habe ich binter der Kollimatorlinse eine Blende eingeschaltet, um nur einen kleinen Flächenteil des Spiegels zu benutzen.

1) A. Wülfing, N. Jahrb. für Miner. Beil.-Bd. 12. 1898. 
Die Lichtstärke wurde hierdurch erheblich vermindert, aber ich war gezwungen dies zu tun, weil die reflektierende Fläche nicht völlig homogen war.

Der Spiegel war mit einer den gewöhnlichen Kristallträgern ähnlichen Vorrichtung auf dem Spektrometer angebracht. Mit drei Stellschrauben und mit Hilfe des Gaussschen Okulars wurde der Spiegel zunächst so justiert, daB seine Fläche genau senkrecht zur horizontalen Drehachse des Trägers stand, und dann wurde diese letzte Drehachse mit andern Stellschrauben parallel zur Einfallsebene gemacht. Alsdann wurde der Träger als ganzes so weit vorwärts oder rückwärts gerückt, dab die Spiegelfäche genau auf den Mittelpunkt des Spektrometers fiel; seine $H o ̈ h e ~ l i e B$ sich gleichfalls justieren, so daB die Drehachse in der Ebene der Fernrohr- und Kollimatorachsen d. h. in der Einfallsebene lag. Die obengenannte Blende hinter dem Kollimator war so eingestellt, daB das kleine Lichtbündel den Spiegel in seinem Mittelpunkt traf. Es ist wichtig, daB man nur ein bestimmtes kleines Gebiet des Spiegels benutzt, weil man nur dann sicher ist, daB irgend eine Verschiedenheit, welche man in verschiedenen Azimuten des Spiegels beobachtet, nicht der Inhomogenität des Spiegels, sondern seiner Anisotropie zuzuschreiben ist. Der Spiegelträger ist mit einem Teilkreise versehen, um das Azimut des Spiegels festzustellen und zu variieren.

\section{Theoretisches.}

Die Theorie der Bestimmung der optischen Konstanten durch Reflexionsmethode ist wohl bekannt. ${ }^{1}$ ) Ich werde hier die Formeln zusammenstellen, die direkt mit den gegenwärtigen Versuchen zusammenhängen und für deren Verständnis nötig sind. Es soll linear polarisiertes Licht, dessen Polarisationsebene unter $\pm 45^{\circ}$ gegen die Einfallsebene geneigt ist, unter dem Einfallswinkel $\varphi$ auf den Spiegel auffallen. Durch die Reflexion an dem Spiegel werden die Amplituden der in der Einfallsebene liegenden $p$-Komponente wie auch die der senkrecht zur Einfallsebene liegenden $s$-Komponente geschwächt und zwar in verschiedenen Verbältnissen. Die Phasen der

1) P. Drude, Lehrb. d. Optik. p. 334. 
Wirkung einer permanenten mechanischen Ausdehnung etc. 813

beiden Komponenten werden auch gegeneinander verschoben, und wir bekommen ein elliptisch polarisiertes Licht. Wenn das Verhältnis der Amplituden der $p$ - und $s$-Komponente des reflektierten Lichtes gleich tang $\psi$ gesetzt wird, und wenn die relative Phasendifferenz der beiden Komponenten durch $\delta$ bezeichnet wird, wobei die $p$-Komponente gegen die $s$-Komponente als beschleunigt angenommen wird, und wenn ferner $\delta=0$ für $\varphi=\pi / 2$, d. h. für streifende Inzidenz und $\delta=\pi / 2$ für $\varphi=0$, d. h. für senkrechte Inzidenz angenommen wird, dann ergeben sich für cinen isotropen Spiegel die Beziehungen

$$
\left\{\begin{aligned}
n & =\sin \varphi \cdot \operatorname{tang} \varphi \cdot \frac{\cos 2 \psi}{1+\cos \delta \cdot \sin 2 \psi} \\
n \varkappa=k & =\sin \varphi \cdot \operatorname{tang} \varphi \cdot \frac{\sin \delta \cdot \sin 2 \psi}{1+\cos \delta \cdot \sin 2 \psi},
\end{aligned}\right.
$$

wobei $n$ den Brechungsexponent, $x$ den Absorptionsindex und $k$ den Absorptionskoeffizient bedeutet.

Für einen Spiegel aus gedehntem Metall ändern sich ebensowohl $\psi$ wie $\delta$ mit dem Azimut $\zeta$ der Spannungsrichtung gegen die Einfallsebene. Hier muß man annehmen, daB der Spiegel sich wie ein einachsiger Kristall verhält. Der Zusammenhang zwischen $\psi, \delta$ und $\zeta$ ist für diesen Fall zuerst von Drude gegeben. ${ }^{1}$ ) Er lautet

$$
\left\{\begin{array}{c}
\frac{\cos 2 \psi}{1-\cos \delta \cdot \sin 2 \psi}+i \frac{\sin \delta \cdot \sin 2 \psi}{1-\cos \delta \cdot \sin 2 \psi} \\
=\frac{\cos ^{2} \zeta\left(\frac{\sqrt{\alpha}}{\cos \varphi}-\sqrt{\beta} \cdot \cos \varphi\right)+\sin ^{2} \zeta\left(\frac{\sqrt{\beta}}{\cos \varphi}-\sqrt{\alpha} \cdot \cos \varphi\right)}{1-(\sqrt{\alpha}-\sqrt{\beta}) \cdot \sin 2 \zeta}
\end{array}\right.
$$

wo $\alpha$ und $\beta$ beide komplexe optische Konstanten sind, und zwar bedeutet

$$
\left\{\begin{array}{l}
\frac{1}{\sqrt{\alpha}}=n_{1}\left(1-i x_{1}\right)=n_{1}-i k_{1} \\
\frac{1}{\sqrt{\beta}}=n_{2}\left(1-i x_{2}\right)=n_{2}-i k_{2} .
\end{array}\right.
$$

Dabei sind $n_{1} n_{2}$ die beiden Hauptbrechungsexponenten und $k_{1} k_{2}$ die beiden Hauptabsorptionskoeffizienten.

1) P. Drude, Wied. Ann. 34. 1888. 
Wir setzen weiter

$$
\left\{\begin{array}{l}
M=\frac{\cos 2 \psi}{1-\cos \delta \cdot \sin 2 \psi}, \\
N=\frac{\sin \delta \cdot \sin 2 \psi}{1-\cos \delta \cdot \sin 2 \psi},
\end{array}\right.
$$

welche beide Funktionen von $\zeta$ sind. Wenn wir die Werte von $M$ und $N$ für $\zeta=0$ und $\zeta=\pi / 2$ bez. durch $M_{1}, N_{1}$ und $M_{2}, N_{2}$ bezeichnen, dann folgt aus den Gleichungen (2) und (3)

$$
\left\{\begin{array}{l}
n_{h}=\sin \varphi \cdot \operatorname{tang} \varphi \frac{M_{h}}{M_{h}^{2}+N_{h}^{2}}, \\
k_{h}=n_{h} x_{h}=\sin \varphi \cdot \operatorname{tang} \varphi \frac{N_{h}}{M_{h}^{2}+N_{h}^{2}},
\end{array} \quad h=1,2 .\right.
$$

Es braucht nicht bemerkt zu werden, daß diese Beziehungen völlig im Einklang mit den Gleichungen (1) stehen.

Wir haben gemäB dem Vorstehenden nach der Beobachtung von $\psi$ und $\delta$ für verschiedene Azimute $\zeta$ zweierlei zu tun. Erst müssen wir ermitteln, ob $M$ und $N$ die Beziehung (2) bez. (4) bestätigen, und dann müssen wir die Hauptkonstanten $n_{h}$ und $k_{h}$ nach (5) bestimmen.

Die Formel (2) lehrt uns eine merkwürdige Tatsache. Wegen des Vorhandenseins des Faktors $\sin 2 \zeta$ im Nenner, sind $\psi$ und $\delta$, und folglich auch $M$ und $N$ nicht symmetrische Funktionen von $\zeta$. Aber dies steht keineswegs im Widerspruch zur angenommenen physikalischen Symmetrie des Spiegels, sondern die Erklärung dafür ist in der unsym. metrischen Lage der Polarisationsebene des einfallenden Lichtes zu finden. ${ }^{1}$ ) Man muB daher berücksichtigen, daß wenn man, um die Variation der optischen Eigenschaften des Spiegels in verschiedenen Azimuten $\zeta$ zu studieren, eine Beobachtungsreihe mit dem Polarisator $+45^{\circ}$ gegen die Einfallsebene mit einer anderen Reihe, in welcher der Polarisator unter $-45^{\circ}$ gegen die Einfallsebene geneigt ist, kombinieren will, man nur die Beobachtung für $\zeta=+a$ und $\zeta=\pi+\alpha$ in der ersten Lage des Polarisators, mit denen für $\zeta=-\alpha$ und $\zeta=-\pi-\alpha$ in der zweiten Lage kombinieren darf.

1) E. C. Müller, N. Jahrb. f. Miner. Beil.-Bd. 17. 1903; F. Pockels, Lehrb. d. Kristalloptik p. 437. 1906. 
Wirkung einer permanenten mechanischen Ausdehnung etc. 815

Der Ausdruck auf der rechten Seite der Gleichung (2) läßt sich folgendermaßen umformen:

und da

$$
\frac{\sin \varphi \cdot \operatorname{tang} \varphi}{2} \cdot \frac{(\sqrt{\alpha}+\sqrt{\beta})+(\sqrt{\alpha}-\sqrt{\beta}) \cos 2 \zeta}{1-(\sqrt{\alpha}-\sqrt{\beta}) \sin 2 \zeta},
$$

$$
\sqrt{\alpha}-\sqrt{\beta} \text { klein gegen } \sqrt{\alpha}+\sqrt{\beta}
$$

ist, kann man ihn annäherungsweise gleich

$$
\frac{\sin \varphi \cdot \operatorname{tang} \varphi}{2}\{(\sqrt{\alpha}+\sqrt{\beta})+(\sqrt{\alpha}-\sqrt{\beta}) \cos 2 \zeta+(\alpha-\beta) \sin 2 \zeta\}
$$

setzen. Durch die Trennung des reellen und des imaginären Bestandteiles erhält man schließlich die Gleichungen von der Form

$$
\left\{\begin{array}{l}
M=P+Q \cos 2 \zeta+R \sin 2 \zeta, \\
N=P^{\prime}+Q^{\prime} \cos 2 \zeta+R^{\prime} \sin 2 \zeta ;
\end{array}\right.
$$

hierbei bestehen, wie leicht zu zeigen ist, unter den reellen Konstanten folgende Beziehungen:

$$
\left\{\begin{array}{l}
R \cdot \sin \varphi \cdot \tan \varphi=2\left(P Q-P^{\prime} Q^{\prime}\right) \\
R^{\prime} \sin \varphi \cdot \operatorname{tang} \varphi=2\left(P Q^{\prime}+P^{\prime} Q\right) .
\end{array}\right.
$$

Bei meinen Versuchen habe ich aus dem beobachteten Wert von $\psi$ und $\delta$ für die verschiedenen $\zeta$ die Konstanten $P, Q, R$ und $P^{\prime}, Q^{\prime}, R^{\prime}$ bestimmt, und daraus die optischen Konstanten berechnet. Aber die Beziehungen (6') konnte ich nicht befriedigend konstatieren, einmal vielleicht weil die Beobachtungen nicht gut genug und sodann weil die oben angenommenen Annäherungen nicht zulässig sind.

\section{Allgemeines über die Beobachtungen.}

Die Bestimmung von $\psi$ und $\delta$ mittels eines Glimmerkompensators und eines Analysators geschieht in bekannter Weise; die ausfübrliche Theorie findet man in den Arbeiten von G. Horn ${ }^{1}$ ) und von E. C. Müller. ${ }^{2}$ ) Wir wollen annehmen, daB das durch die Reflexion hervorgerufene elliptischpolarisierte Licht seine große Achse um einen Winkel $\Theta$ gegen

1) G. Horn, N. Jahrb. f. Miner. Beil.-Bd. 12. 1899.

2) E. C. Müller, l. e. 
die Einfallsebene geneigt hat, und sein Achsenverhältnis gleich tang $J$ ist, dann ergibt sich

$$
\left\{\begin{array}{l}
\cos 2 \psi=\cos 2 J \cdot \cos 2 \Theta, \\
\operatorname{tang} \delta=\frac{\tan 2 J}{\sin 2 \Theta} .
\end{array}\right.
$$

$J$ und $\Theta$ werden direkt aus den Ablesungen am Kompensator und am Analysator berechnet.

Wenn der Kompensator eine gewisse geeignete Lage einnimmt, wird sich das elliptisch polarisierte Licht wieder in ein linear polarisiertes Licht verwandeln, und daher wird es uns möglich, durch die Drehung des Analysators das Gesichtsfeld völlig dunkel zu machen. Wo dies der Fall ist, gibt es zwei solcher Lagen des Kompensators und dementsprechend zwei Lagen des Analysators. Diese Lagen für den Kompensator mögen durch Winkel $\xi_{1}$ und $\xi_{2}$ bestimmt sein. Diese Winkel liegen zwischen der Polarisationsebene der langsameren Welle im Glimmer und der großen Achse der Ellipse. Wenn die entsprechenden Ablesungen des Kompensators bez. des Analysators durch $c_{1}, c_{2}$ bez. $a_{1}, a_{3}$ bezeichnet werden, so haben wir

und

$$
\begin{aligned}
& \left\{\begin{array}{l}
\xi_{1}-\xi_{2}=c_{1}-c_{2}, \\
\eta_{1}-\eta_{2}=\left(a_{1}-a_{2}\right)-\left(c_{1}-c_{2}\right),
\end{array}\right. \\
2 \theta & =\left(a_{1}+a_{2}\right)-2 a_{0}+\pi \\
= & \left.\left(c_{1}+c_{2}\right)-2 c_{0}+\frac{\pi}{2}\right\} \text { wenn } \Delta<\frac{\pi}{2} \\
= & \left(a_{1}+a_{2}\right)-2 a_{0} \\
= & \left.\left(c_{1}+c_{2}\right)-2 c_{0}-\frac{\pi}{2}\right\} \text { wenn } \Delta>\frac{\pi}{2},
\end{aligned}
$$

$$
\left\{\begin{array}{l}
\cos 2 J=\frac{\sin \left(\eta_{1}-\eta_{2}\right)}{\sin \left(\xi_{1}-\xi_{2}\right)}, \\
\operatorname{tang} \Delta= \pm \frac{\operatorname{tang} 2 J}{\cos \left(\xi_{1}-\xi_{2}\right)}, \quad+\text { wenn } \Delta<\frac{\pi}{2},
\end{array}\right.
$$

wobei $\Delta$ die Phasendifferenz der zwei Wellen im Glimmer ist.

Wenn $\Delta$ des Kompensators für die benutzte Lichtart bekannt ist, braucht man nicht $a_{1}$ und $a_{2}$ abzulesen. Aber ich habe es rorgezogen, durch die Ablesungen des Kompensators und des Analysators den Wert des zu einer Serie der Messungen 
gehörenden $\Delta \mathrm{zu}$ berechnen und das arithmetische Mittel zu nehmen; dieser Mittelwert des $\Delta$ ist benutzt für die Berechnung des $J$. Für $\Theta$ habe ich immer das Mittel von den zwei Werten aus $c_{1}, c_{2}$ und aus $a_{1}, a_{2}$ genommen.

In der Tab. I ist der Wert rom $\Delta$ angegeben mit dem wahrscheinlichen Fehler $\varepsilon$ und dem Termin der Beobachtung. Ob die ziemlich große Änderung des $\Delta$ in dem Kompensator I im Monat Juni der Temperaturschwankung oder einer anderen Ursache zuzuschreiben ist, kann ich nicht bestimmt sagen.

Tabelle I.

Phasendifferenz $\Delta$ des Kompensators für D-Licht.

\begin{tabular}{|c|c|c|c|c|}
\hline & $\Delta$ & & $\varepsilon$ & Datum \\
\hline 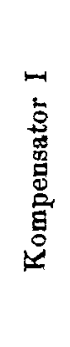 & 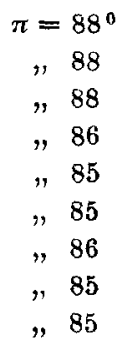 & $\begin{array}{r}41,4^{r} \\
30,5 \\
1,0 \\
3,9 \\
54,2 \\
51,3 \\
2,3 \\
52,1 \\
29,9\end{array}$ & $\begin{array}{l}2,7^{\prime} \\
4,4 \\
3,3 \\
3,6 \\
2,6 \\
4,3 \\
1,4 \\
3,9 \\
2,7\end{array}$ & $\begin{array}{l}\text { 8.-11. April } \\
\text { 14.-16. Mai } \\
\text { 30. Mai-2. Juni } \\
\text { 13.-14. Juni } \\
\text { 21.-22. " } \\
\text { 29. Juli-3. Aug. } \\
\text { 4.-10. August } \\
\text { 7.-13 " } \\
\text { 18.-27. Nov. }\end{array}$ \\
\hline 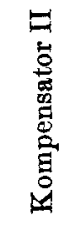 & $\begin{array}{r}\pi=83^{0} \\
", \\
", \\
", \\
", \\
", \\
",\end{array}$ & $\begin{array}{l}34,4 \\
15,4 \\
15,1 \\
43,8 \\
44,1 \\
50,0\end{array}$ & $\begin{array}{r}5,9^{\prime} \\
1,6 \\
10,0 \\
9,0 \\
2,1 \\
10,5\end{array}$ & $\begin{array}{l}\text { 22. Juni } \\
\text { 28. " } \\
\text { 29. Juni - 1; Juli } \\
\text { 8. Juli } \\
\text { 17.-27. Juli } \\
\text { 1.-4. August }\end{array}$ \\
\hline
\end{tabular}

Die Beobachtungsresultate.

Ich werde jetzt zu der Beschreibung der einzelnen Ver. suche übergehen. Die von mir untersuchten Metalle sind Kupfer, Silber und Stahl. Sämtliche Spiegel sind von Schmidt und Haensch poliert.

\section{Kupfer.}

1. Spannungsfreies Kupfer.

Die Beobachtung ist mit dem Spektrometer II gemacht. Der Polarisator ist in vier Lagen eingestellt, und es sind jedesmal 20 Ablesungen von $c_{1}, c_{2}$ und $a_{1}, a_{2}$ genommen worden. 
Es möge hier eine Reihe solcher Ablesungen als ein Beispiel wiedergegeben werden, um die Genauigkeit der einzelnen Einstellungen $\mathrm{zu}$ zeigen. Der Nullpunkt des Polarisators ist $p_{0}=79^{\circ} 32^{\prime}$, und seine Einstellung bei der Messung war $304^{0} 32^{\prime}$; das einfallende Licht ist also wie $\swarrow^{\nearrow}$ polarisiert.

\section{Tabelle II.}

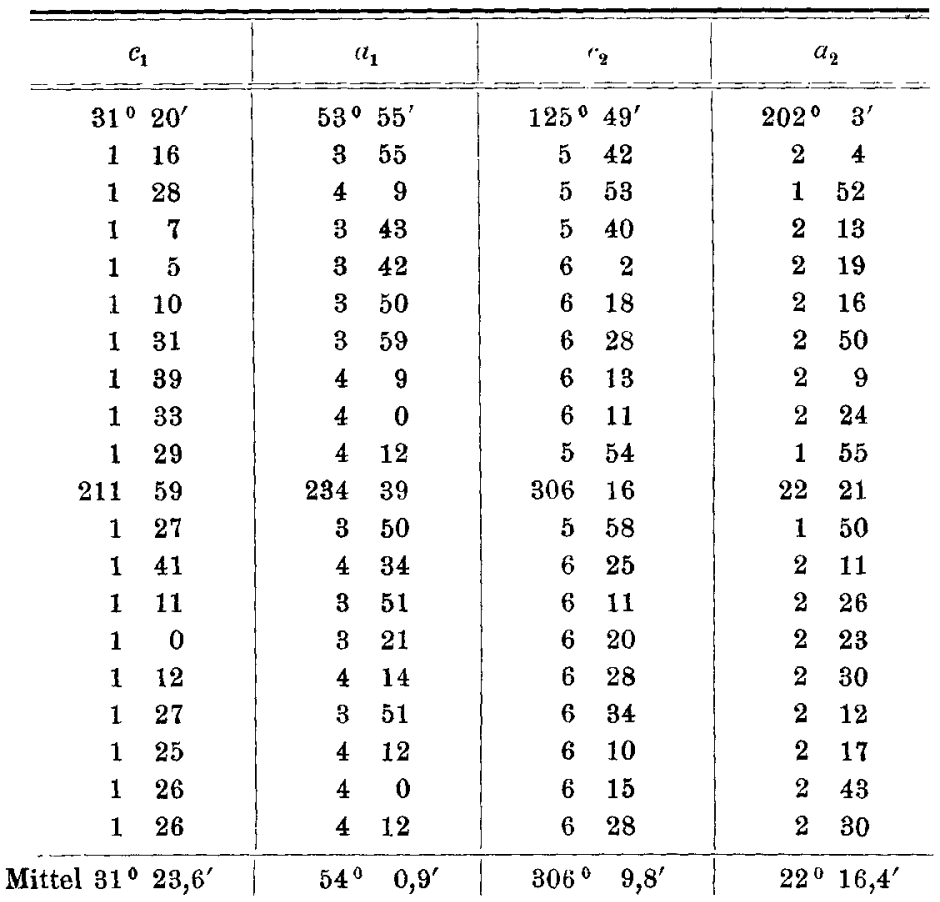

Der Mittelwert für die vier Lagen des Polarisators ist:

Tabelle III.

\begin{tabular}{|c|c|c|c|c|c|c|}
\hline $\begin{array}{c}\text { Polari- } \\
\text { sator }\end{array}$ & $\begin{array}{c}\text { Einfallendes } \\
\text { Licht }\end{array}$ & $c_{1}$ & $c_{2}$ & $a_{1}$ & $a_{2}$ & $c_{0}=87^{0} \quad 7^{\prime}$ \\
\hline $304^{0} 32^{\prime}$ & & $31^{0} 24^{\prime}$ & $306^{\circ} 10^{\prime}$ & $54^{0} \mathrm{I}^{\prime}$ & $22^{0} 16^{\prime}$ & $a_{0}=9134$ \\
\hline 21432 & & 14231 & 22754 & 12846 & 16038 & $\varphi=45$ \\
\hline 12432 & & 3337 & $308 \quad 29$ & 5459 & 2625 & Ten \\
\hline 3432 & 7 & $140 \quad 0$ & $225 \quad 36$ & 12817 & $156 \quad 44$ & \\
\hline
\end{tabular}


Wirkung einer permanenten mechanischen Ausdelnung etc.

Daraus folgt

$$
\begin{aligned}
\xi_{1}-\xi_{2} & =85^{\circ} 20^{\prime} & & n_{0}=0,7083 \\
\eta_{1}-\eta_{2} & =55 \quad 11 & & k_{0}=n_{0} x_{0}=1,9904 \\
2 \Theta & =75 \quad 52 & & x_{0}=2,815 . \\
2 J & =34 \quad 30 & & \\
2 \psi & =78 \quad 23 & & \\
\delta & =\pi-35^{0} 20^{\prime} & &
\end{aligned}
$$

Der Index 0 soll bedeuten, daß die betreffende Größe zum spannungsfreien Zustand gehört.

Die Konstanten für Kupfer, gefunden von Drude mittels eines Babinetschen Kompensators, sind

$$
\begin{aligned}
n_{0} & =0,64, \\
n_{0} x_{0} & =2,62, \\
x_{0} & =4,10 .
\end{aligned}
$$

Nach Drude sind die Wirkungen einer Oberflächenschicht und einer matten Politur die folgenden: Wenn man den Einfallswinkel, für welchen $\delta=\pi / 2$ beträgt durch $\phi$ und den dementsprechenden Wert von $\psi$ durch $\bar{\psi}$ bezeichnet, dann verkleinert die Oberflächenschichte $\bar{\varphi}$ und vergrößert $\bar{\psi}$; die matte Politur verkleinert $\bar{\varphi}$ ein weniy und $\bar{\psi}$ bedeutend. Man hat die Beziehungen

otder

$$
x=\operatorname{tang} 2 \bar{\psi},
$$

$$
\sin \bar{\varphi} \cdot \operatorname{tang} \bar{\varphi}=n \sqrt{1+\varkappa^{2}}=n \sec 2 \bar{\psi},
$$

$$
n=\sin \varphi \cdot \tan g \cdot \hat{\varphi} \cdot \cos 2 \bar{\psi} .
$$

Durch Beobachtung eines Spiegels mit einer Oberfächenscbichte bekommen wir also zu großes $\varkappa$ und zu kleines $n$; und mit einem Spiegel matter Politur bekommen wir zu kleines $x$ und vielleicht zu großes $n$. Wenn man die Drudeschen Zahlen zugrunde legt und gleiche Substanz voraussetzt, würde mein Spiegel nicht blank genug gewesen sein.

2. Gedehntes Kupfer.

Der oben benutzte Spiegel wurde gedehnt. Aber durch den Zug war das Metall geflossen und seine Fläche sehr schlecht geworden. Ich habe mir viele Mühe gegeben, um einige brauchbare Ablesungen zu erhalten, aber es kam schließ- 
lich heraus, daB sie zu unzuverlässig waren and ich sie verwerfen muBte.

Mit einem anderen Spiegel, der erst gedehnt und dann poliert war, konnte ich eine Reihe Ablesungen machen. Das Spektrometer I wurde benutzt. Der Polarisator war eingestellt in zwei Lagen $+45^{\circ}$ und $-45^{\circ}$, und in jeder dieser Lagen wurden je acht Ablesungen für jede der acht Lagen des Spiegelträgers gemacht. Die Ablesungen des Teilkreises des Trägers sind

$$
A=0^{\circ}, 45^{\circ}, 90^{\circ}, 135^{\circ}, 180^{\circ}, 225^{\circ}, 270^{\circ}, 315^{\circ} \text {. }
$$

Da bei $A_{0}=110^{\circ}$ die Spannungsrichtung des Spiegels der Einfallsebene parallel war, so entsprechen diese Lagen

$$
\zeta=250^{\circ}, 295^{\circ}, 340^{\circ}, 25^{\circ}, 70^{\circ}, 115^{\circ}, 160^{\circ}, 205^{\circ} \text {. }
$$

Wenn man die Beobachtung auf die Lage $+45^{\circ}$ des Polarisators reduzieren will, darf man nicht ohne weiteres die Zahlen für die beiden Lagen kombinieren. Wie ich schon früher hervorgehoben habe, ist $\zeta=+\alpha$ für die Lage $-45^{\circ}$ gleich. wertig mit $\zeta=-\infty$ für die Lage $+45^{\circ}$. Auf die Lage $+45^{0}$ reduziert, sind also

$$
A=0^{\circ}, 45^{\circ}, 90^{\circ}, 135^{\circ}, 180^{\circ}, 225^{\circ}, 270^{\circ}, 315^{\circ}
$$

für $+45^{0}$ gleichwertig mit

$$
\zeta=250^{\circ}, 295^{\circ}, 340^{\circ}, 25^{\circ}, 70^{\circ}, 115^{\circ}, 160^{\circ}, 205^{\circ} \text {, }
$$

aber für $-45^{\circ}$ sind sie gleichwertig mit

$$
\zeta=110^{\circ}, 65^{\circ}, 20^{\circ}, 335^{\circ}, 290^{\circ}, 245^{\circ}, 200^{\circ}, 155^{\circ} \text {. }
$$

Die Tab. IV ist konstruiert aus dem Mittelwert der acht Ablesungen für die acht Azimute des Spiegels in den beiden Lagen des Polarisator. $\zeta$ bezieht sich auf die Lage $+45^{\circ}$ des Polarisators.

$$
\text { Tabelle IV. }
$$

Gedehntes Kupfer, $\frac{\Delta l}{l}=0,026$.

$$
\varphi=75^{\circ} \text {. }
$$

\begin{tabular}{c|c|c|c|c|c}
\hline \hline Pol. & $A$ & $\zeta$ & $\xi_{1}-\xi_{2}$ & $\eta_{1}-\eta_{2}$ & $2 \Theta$ \\
\hline \hline$+45^{0}$ & $0^{0}-180^{\circ}$ & 70 & $87^{\circ} 58^{\prime}$ & $39^{\circ} 38^{\prime}$ & $61^{\circ} 2^{\prime}$ \\
$\swarrow^{\nearrow}$ & $45-225$ & 115 & 887 & 3848 & 6134 \\
& $90-270$ & 160 & 8744 & 3836 & 6125 \\
& $135-315$ & 25 & 8816 & 3929 & 6119
\end{tabular}


Tabelle IV (Fortsetzung).

\begin{tabular}{c|c|c|c|c|c}
\hline \hline Pol. & $A$ & $\zeta$ & $\xi_{1}-\xi_{2}$ & $\eta_{1}-\eta_{2}$ & $2 \Theta$ \\
\hline \hline$-45^{0}$ & $0^{0}-180^{\circ}$ & 110 & $88^{\circ} 34^{\prime}$ & $38^{\circ} 30^{\prime}$ & $60^{\circ} 29^{\prime}$ \\
$K_{\searrow}$ & $45-225$ & 65 & 8810 & 3735 & 614 \\
& $90-270$ & 20 & 8730 & 3720 & 6047 \\
& $135-315$ & 155 & 8814 & 3815 & 61 \\
\end{tabular}

Daraus erhalten wir:

Tabelle $\nabla$.

\begin{tabular}{|c|c|c|c|c|c|c|c|c|}
\hline$\zeta$ & $2 \psi$ & $\delta$ & $M_{\text {beob. }}$ & $M_{\text {ber. }}$ & Diff. & $N_{\text {beob. }}$ & $N_{\text {ber. }}$ & Diff. \\
\hline $20^{\circ}$ & $72^{\circ} 46^{r}$ & $56^{0} 19^{\prime}$ & 0,630 & 0,658 & $-0,028$ & 1,690 & 1,719 & $-0,029$ \\
\hline 25 & 7213 & 547 & 691 & 659 & 32 & 1,747 & 1,719 & 28 \\
\hline 65 & 7250 & $\begin{array}{ll}56 & 1\end{array}$ & 634 & 669 & 35 & 1,700 & 1,722 & 22 \\
\hline 70 & $\begin{array}{ll}72 & 0\end{array}$ & $54 \quad 2$ & 700 & 669 & 31 & 1,745 & 1,722 & 23 \\
\hline 110 & 728 & $\begin{array}{ll}55 & 19\end{array}$ & 669 & 668 & + & 1,707 & 723 & 16 \\
\hline 115 & 7238 & $54 \quad 42$ & 665 & 667 & - & 36 & & 13 \\
\hline 155 & $72 \quad 32$ & 5523 & 655 & 658 & - & 1,713 & 1,720 & - \\
\hline 160 & $72 \quad 37$ & 5456 & 661 & 657 & + & 1,730 & 1,720 & 10 \\
\hline
\end{tabular}

Aus dem beobachteten Wert des $M$ und $N$ bekommen wir durch die Methode der kleinsten Quadrate

$$
\left.\begin{array}{l}
M=0,6632-0,0074 \cdot \cos 2 \zeta+0,0009 \cdot \sin 2 \zeta \\
N=1,721-0,0022 \cdot \cos 2 \zeta-0,0007 \cdot \sin 2 \zeta
\end{array}\right\} \varphi=75^{\circ},
$$

daraus erhalten wir für $\zeta=0$ und für $\zeta=\pi / 2$

$$
\begin{aligned}
M_{\|} & =0,6558, & M_{\perp} & =0,6706, \\
N_{\|} & =1,719, & N_{\perp} & =1,723, \\
n_{\|} & =0,6982, & n_{\perp} & =0,7073, \\
k_{\|} & =1,830, & k_{\perp} & =1,817, \\
\varkappa_{\|} & =2,621, & \varkappa_{\perp} & =2,569 .
\end{aligned}
$$

Die Dehnung des Spiegels ist mit einem Komparator gemessen und sie beträgt

$$
\frac{\Delta l}{l}=0,026 \text {. }
$$

Da die Differenzen zwischen den beobachteten und den berechneten. Werten des $M$ und $N$ ziemlich groB sind, habe ich 
die Beobachtung wiederholt unter demselben Zustande des Apparates. Es wurde in beiden Lagen des Polarisators beobachtet.

Tabelle VI.

Ausgedehntes Kupfer, $\frac{\Delta l}{l}=0,026$.

$\varphi=75^{\circ}$.

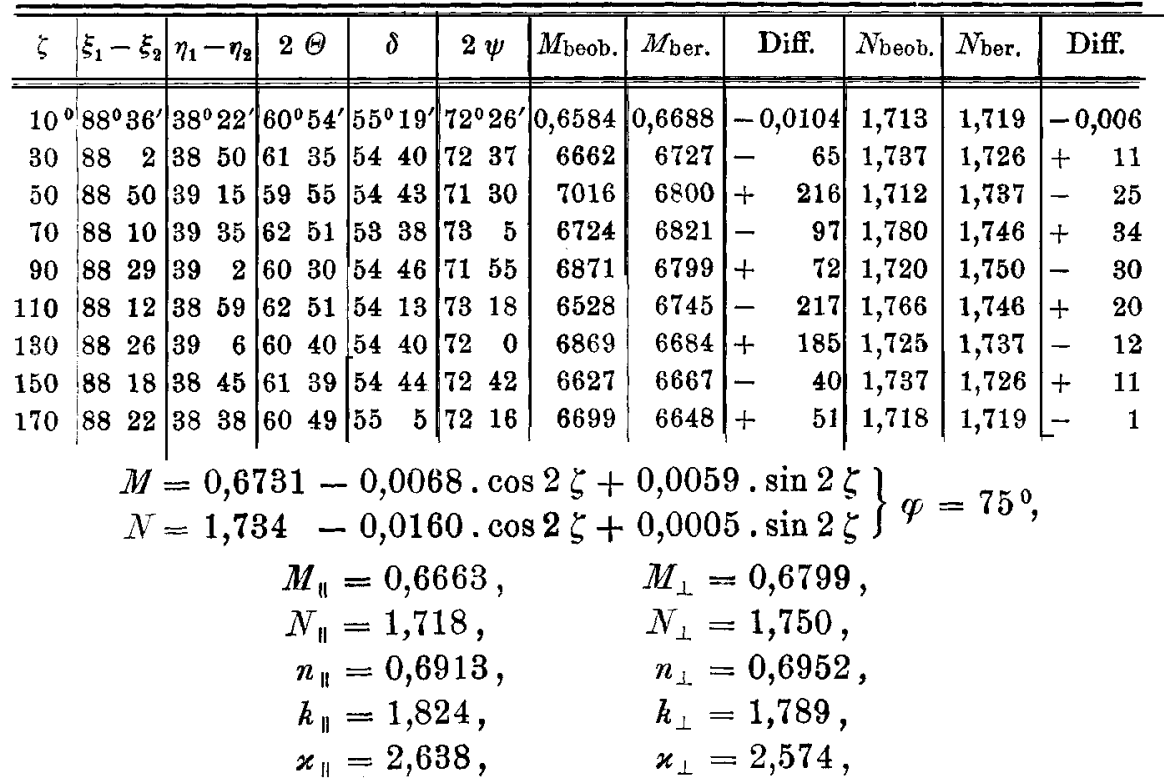

Trotzdem die einzelnen Beobachtungsreihen in sich nicht gut stimmen, fallen die aus ihnen folgenden Parameter leidlich zusammen, so daB über den Sinn der Anisotropie kein $Z$ weifel bleibt.

\section{Silber.}

Bei Silber hatte ich das Glück, mit demselben Stück die beiden Versuchsreihen ausführen zu können. Es wurde ausschlieBlich mit dem Spektrometer II beobachtet. Für den spannungsfreien Zustand habe ich 20 Ablesungen des $c_{1}, c_{2}$ und des $a_{1}, a_{2}$ für jede der vier Lagen des Polarisators $g e$ nommen. Als den Mittelwert für $\varphi=45^{\circ}$ habe ich gefunden:

$$
\begin{array}{rlrl}
\xi_{1}-\xi_{2} & =87^{\circ} 3^{\prime} & 2 J & =23^{0} 28^{\prime} \\
\eta_{1}-\eta_{2} & =6622 & 2 \psi & =88 \quad 1 \\
2 \Theta & =8750 & \delta & =\pi-23^{\circ} 28^{\prime} .
\end{array}
$$


Wirkung einer permanenten mechanischen Ausdehnung etc. 823

Daraus folgt

$$
\left.\begin{array}{l}
n_{0}=0,2934 \\
k_{0}=3,368 \\
x_{0}=11,48
\end{array}\right\} \text { Temp. } 22^{0} .
$$

Nach Drude gilt

$$
\begin{aligned}
& n_{0}=0,18, \\
& k_{0}=3,67, \\
& \varkappa_{0}=20,39,
\end{aligned}
$$

Die Differenz weist, bei Voraussetzung gleichen Materiales, wieder darauf hin, daß mein Spiegel wahrscheinlich nicht blank genug war.

Das Metallstiuck wurde gedehnt, und die Elongation $\Delta l / l$ gemessen; sie betrug 0,0027. An einigen Stellen der Fläche konnte man deutlich die Unebenheit des Spiegels sehen, aber im ganzen war die Fläche ziemlich gut geblieben. Die Stelle, wo die Fläche am besten aussah, wurde auf der Verlängerung der Druckachse des Spiegelträgers gebracht, und mit Hilfe der Blende konnte ich den folgenden Versuch ausführen. Der Polarisator wurde in seinen vier Lagen eingestellt, und für jede Lage des Polarisators und des Spiegels wurden vier $\mathbf{A b}$ lesungen gemacht.

\begin{tabular}{|c|c|c|c|c|c|c|c|c|c|c|c|}
\hline$\zeta$ & $\xi_{1}-\xi_{2}$ & $\eta_{1}-\eta_{2}$ & $2 \Theta$ & $-\delta$ & $2 \psi$ & I beob. & Vber. & Diff. & $N_{\text {beob. }}$ & $N_{\text {ber. }}$ & Diff. \\
\hline $0^{\circ}$ & ${ }^{0} 16^{\prime}$ & $18^{\circ} 58^{\prime}$ & $80^{\circ} 10^{\prime}$ & $70^{\circ} 27$ & $86^{\circ} 41^{\prime}$ & 0,0434 & 0,0475 & $-0,0041$ & & 0,7087 & 036 \\
\hline 30 & 7155 & 1754 & 7732 & 7057 & 8552 & 543 & 519 & 24 & 03 & 7145 & 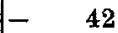 \\
\hline 60 & 7053 & $\begin{array}{ll}17 & 53\end{array}$ & $73 \quad 47$ & 720 & 454 & & 31 & - & 5 & 09 & + \\
\hline 90 & $71 \quad 42$ & $\begin{array}{ll}18 & 13\end{array}$ & 7140 & 7138 & $84 \quad 2$ & & 799 & - & & 215 & 29 \\
\hline & $72 \quad 5$ & 1858 & 7255 & $71 \quad 10$ & 8420 & 747 & 755 & - & 8 & 157 & 29 \\
\hline 50 & $\left|\begin{array}{ll}72 & 46\end{array}\right|$ & $\left|\begin{array}{ll}19 & 8\end{array}\right|$ & $76 \quad 4$ & 17013 & $85 \quad 12$ & 626 & 593 & + & 7176 & 7093 & + \\
\hline
\end{tabular}

\section{Tabelle VII.}

Gedehntes Silber, $\frac{A l}{l}=0,0027$.

$\varphi=70^{\circ}$.

$$
\left.\begin{array}{l}
M=0,0637-0,0162 \cdot \cos 2 \zeta-0,0043 \cdot \sin 2 \zeta \\
N=0,7151-0,0064 \cdot \cos 2 \zeta+0,0028 \cdot \sin 2 \zeta
\end{array}\right\} \varphi=70^{\circ} .
$$


Wir haben daraus

$$
\begin{aligned}
& M_{\|}=0,0475, \\
& N_{\text {॥ }}=0,7087 \text {, } \\
& \left.\begin{array}{l}
M_{\perp}=0,0799 \\
N_{\perp}=0,7215
\end{array}\right\} \varphi=70^{\circ}, \\
& n_{\text {॥ }}=0,2431 \text {, } \\
& n_{\perp}=0,3915 \text {, } \\
& k_{\|}=3,627 \text {, } \\
& k_{\perp}=3,535 \text {, } \\
& x_{\|}=14,92 \text {, } \\
& \varkappa_{\perp}=9,03 \text {. }
\end{aligned}
$$

\section{Stahl.}

Bei Stahl konnte ich mit fünf Spiegeln arbeiten, welche ich durch die Buchstaben $A, B, C, D, E$ voneinander unter-

\begin{tabular}{c|c}
\hline \hline Spiegel & $\frac{\Delta l}{l}$ \\
\hline \hline$A$ & 0,021 \\
$B$ & 0,108 \\
$C$ & 0,055 \\
$D$ & 0,013
\end{tabular}
schieden habe. Der Spiegel $E$ ist spannungsfrei, die anderen sind aus gedehntem Metall, und zwar sind sie nach der Elongation poliert worden. Die Größe der Elongationen sind in der nebenstehenden kleinen Tabelle gegeben.

1. Spannungsfreier Stahlspiegel $E$.

\begin{tabular}{|c|c|c|c|c|c|c|c|c|c|c|}
\hline$\varphi$ & $\xi_{1}-\xi_{2}$ & $\eta_{1}-\eta_{2}$ & $2 \theta$ & $2 J$ & $\Delta-x$ & $2 \psi$ & $\delta$ & $n$ & $k$ & $x$ \\
\hline 30 & $89^{0} 38^{\prime}$ & $76^{\circ} 57^{\prime}$ & $-81^{\circ} 8^{\prime}$ & $13^{0} 2^{\prime}$ & $88^{\circ} 25^{\prime}$ & $81^{\circ} 22^{\prime}$ & $13^{0} 12^{\prime}$ & 2,187 & 3,287 & 1,50 \\
\hline 5 & $\begin{array}{ll}89 & 11\end{array}$ & $\begin{array}{ll}59 & 3\end{array}$ & $\begin{array}{ll}-65 & 4\end{array}$ & $\begin{array}{ll}30 & 56\end{array}$ & $88 \quad 38$ & $\begin{array}{ll}68 & 48\end{array}$ & 4123 & 423 & 4,129 & 705 \\
\hline 30 & $\begin{array}{ll}88 & 22\end{array}$ & $40 \quad 30$ & $\begin{array}{ll}-30 & 11\end{array}$ & 4929 & 8836 & $55 \quad 50$ & 6645 & 350 & 3,181 & 854 \\
\hline 7 & $87 \quad 54$ & $36 \quad 0$ & -645 & $\begin{array}{ll}53 & 58\end{array}$ & 8828 & $54 \quad 16$ & 857 & 306 & 3,193 & 385 \\
\hline 6 & 8739 & $36 \quad 32$ & +230 & $\begin{array}{ll}53 & 26\end{array}$ & $\begin{array}{ll}88 & 15\end{array}$ & $\begin{array}{ll}53 & 28\end{array}$ & 9151 & 378 & 3,209 & 349 \\
\hline 5 & 8836 & $\begin{array}{ll}36 & 30\end{array}$ & +128 & $\begin{array}{ll}53 & 29\end{array}$ & $\begin{array}{ll}88 & 58\end{array}$ & $54 \quad 26$ & 9851 & 397 & 3,311 & 382 \\
\hline 0 & $87 \quad 46$ & 447 & +3637 & 4550 & 8750 & $56 \quad 0$ & $120 \quad 5$ & 470 & 3,169 & 283 \\
\hline$\theta$ & 897 & 5150 & +5251 & $\begin{array}{ll}38 & 9\end{array}$ & $88 \quad 52$ & 6139 & 13525 & 474 & 3,218 & 301 \\
\hline \multicolumn{2}{|c|}{ Mittel } & & & & $88^{\circ} 30$ & & & 373 & 330 & 395 \\
\hline
\end{tabular}

Da der Spiegel $E$ scheinbar sehr gut poliert war, habe ich versucht, in verschiedenen Einfallswinkeln zu beobachten, um zu sehen, ob die Gleichungen (1) mit dem Versuch übereinstimmen. Der Spektrometer I wurde benutzt, dessen Polarisator immer in der Lage $-45^{\circ}$ eingestellt blieb. Vier $\mathrm{Ab}$ lesungen sind gemacht für verschiedene $\varphi$.

Tabelle VIII.

Spannungsfreier Stahl. 
Wirkung einer permanenten mechanischen Ausdehnung etc. 825

Durch den glatten Verlauf des $\psi$ und $\delta$, und durch die gute Übereinstimmung $\operatorname{der} n, k$ und $x$ untereinander (ausgenommen die Zahlen für $\varphi=88^{\circ}, 85^{\circ}$ ) sind die Gleichungen (1) konstatiert. Wir erhalten also den Mittelwert

$$
n_{0}=2,373, \quad k_{0}=3,337, \quad x_{0}=1,395 .
$$

Drude erhielt $n_{0}=2,41, k_{0}=3,40, x_{0}=1,41$.

2. Gedehnter Stahlspiegel $D$.

Der Spiegel ist eingestellt für $\zeta=0^{\circ}, 90^{\circ}, 180^{\circ}, 270^{\circ}$, mit dem Einfallswinkel $\varphi$ gleich $77^{\circ}$, und der Polarisator für seine vier Lagen. Vier Ablesungen wurden jedesmal genommen. Das Resultat ist in der Tabelle gegeben.

$$
\begin{gathered}
\text { Tabelle IX. } \\
\text { Gedehnter Stahl, } \frac{\Delta l}{l}=0,013 . \\
\varphi=77^{\circ} .
\end{gathered}
$$

\begin{tabular}{c|c|c}
\hline \hline & $\|$ & $\perp$ \\
\hline \hline$\xi_{1}-\xi_{9}$ & $84^{0} 53^{\prime}$ & $85^{0} 19^{\prime}$ \\
$\eta_{1}-\eta_{2}$ & $38 \quad 6$ & 3934 \\
$2 \Theta$ & 747 & $9 \quad 1$ \\
$2 \psi$ & 528 & 5051 \\
$\delta$ & 8354 & 8238 \\
$n$ & 2,390 & 2,424 \\
$k$ & 3,056 & 2,953 \\
$\varkappa$ & 1,279 & 1,219
\end{tabular}

Um die Variationen von $\psi$ und $\delta$ für verschiedene Azimute des Spiegels zu studieren, wurde der folgende Versuch mit demselben Spiegel gemacht. Der Einfallswinkel $\varphi$ war, wie früher, $77^{\circ}$ und der Polarisator wurde eingestellt in seinen vier Lagen. Die Spannungsrichtung im Spiegel war parallel zur Einfallsebene, wenn die Ablesung des Spiegelträgers $A_{0}=110^{\circ}$ war, und da die sukzessiven Lagen des Trägers

$$
A=0^{\circ}, 30^{\circ}, 60^{\circ}, 90^{\circ}, 120^{\circ}, 150^{\circ}, \ldots 330^{\circ}
$$

waren, wenn wir die Beobachtungen auf die $\swarrow^{\nearrow}$ Lage des Polarisators reduzieren, entsprachen sie

$$
\zeta=70^{\circ}, 100^{\circ}, 130^{\circ}, 160^{\circ}, 10^{\circ}, 40^{\circ},
$$


für die $\swarrow^{\nearrow}$ Lage, während für die $\nwarrow_{\searrow}$ Lage sie entsprachen

$$
\zeta=110^{\circ}, 80^{\circ}, 50^{\circ}, 20^{\circ}, 170^{\circ}, 140^{\circ}
$$

Vier Ablesungen wurden jedesmal genommen.

\section{Tabelle X.}

Gedehnter Stahl, $\frac{\Delta l}{l}=0,013$.

$$
q=77^{\circ} \text {. }
$$

\begin{tabular}{|c|c|c|c|c|c|}
\hline Pol. & A & $\zeta$ & $\xi_{1}-\xi_{2}$ & $\eta_{1}-\eta_{2}$ & $2 \Theta$ \\
\hline \multirow{6}{*}{$k^{\lambda}$} & $0^{\circ}-180^{\circ}$ & $70^{\circ}$ & $87^{0} 53^{\prime}$ & $39^{\circ} 42^{\prime}$ & $8^{0} 52^{\prime}$ \\
\hline & $30-210$ & 100 & 8758 & $\begin{array}{ll}39 & 12\end{array}$ & 821 \\
\hline & $60-240$ & 130 & 8836 & 3928 & 850 \\
\hline & $90-270$ & 160 & 8836 & $38 \quad 44$ & 831 \\
\hline & $120-300$ & 10 & $\begin{array}{ll}88 & 17\end{array}$ & $38 \quad 35$ & 754 \\
\hline & $150-330$ & 40 & $\begin{array}{ll}88 & 7\end{array}$ & 3922 & 756 \\
\hline \multirow{6}{*}{$x_{x}$} & $0^{\circ}-180^{\circ}$ & $110^{\circ}$ & $86^{0} 32^{\prime}$ & $39^{\circ} 44^{\prime}$ & $8^{\circ} 50^{\prime}$ \\
\hline & $30-210$ & 80 & $\begin{array}{ll}86 & 57\end{array}$ & 3950 & 88 \\
\hline & $60-240$ & 50 & 8631 & $39 \quad 0$ & 740 \\
\hline & $90-270$ & 20 & $86 \quad 54$ & $\begin{array}{ll}38 \quad 12\end{array}$ & 736 \\
\hline & $120-300$ & 170 & $87 \quad 3$ & 3836 & 82 \\
\hline & $150-330$ & 140 & $87 \quad 5$ & $\begin{array}{ll}39 & 12\end{array}$ & 759 \\
\hline
\end{tabular}

Daraus erhalten wir:

\begin{tabular}{|c|c|c|c|c|c|c|c|c|}
\hline$\zeta$ & $2 \psi$ & $\delta$ & $M_{\text {beob. }}$ & $M_{\text {ber. }}$ & Diff. & $N_{\text {beob. }}$ & $N$ ber. & Diff. \\
\hline $10^{\circ}$ & $51^{\circ} 49^{\prime}$ & $83^{\circ} 44^{\prime}$ & 0,6620 & 0,6715 & $-0,0095$ & 0,8542 & 0,8538 & $+0,0004$ \\
\hline 20 & 527 & $84 \quad 3$ & 6686 & 6727 & 39 & 8548 & 8524 & 24 \\
\hline 40 & 513 & 8332 & 6889 & 6797 & 92 & 8470 & 8497 & 27 \\
\hline 50 & $51 \quad 19$ & 8357 & 6810 & 6845 & 35 & 8460 & 8487 & 27 \\
\hline 70 & 5050 & 8242 & 7005 & 6943 & 62 & 8532 & 8478 & 54 \\
\hline 80 & 5034 & 8315 & 6983 & 6979 & + & 8437 & 8482 & 45 \\
\hline 100 & $51 \quad 16$ & 8314 & 6890 & 7007 & 117 & 8530 & 8500 & 30 \\
\hline 110 & $50 \quad 45$ & 8242 & 7018 & 6995 & 23 & 8522 & 8514 & + \\
\hline 130 & $51 \quad \check{5}$ & 8247 & 6963 & 6925 & 38 & 8554 & 8541 & 13 \\
\hline 140 & $51 \quad 11$ & 8331 & 6872 & 6877 & $\rightarrow$ & 8487 & 8551 & - \\
\hline 160 & 5145 & 8312 & 6824 & 6779 & 45 & 8597 & 8560 & 37 \\
\hline 170 & 5147 & $83 \quad 37$ & 6778 & 6743 & 35 & 8555 & 8556 & 1 \\
\hline
\end{tabular}

Tabelle XI. 
Hirkung einer permanenten magnetischen Ausdehnung etc.

$$
\begin{array}{cc}
M=0,6861-0,0140 \cdot \cos 2 \zeta-0,0041 \cdot \sin 2 \zeta, \\
N=0,8519+0,0030 \cdot \cos 2 \zeta-0,0028 \cdot \sin 2 \zeta, \\
M_{\|}=0,6721, & M_{\perp}=0,7001, \\
N_{\|}=0,8549, & N_{\perp}=0,8489, \\
n_{\|}=2,399, & n_{\perp}=2,441, \\
k_{\|}=3,052, & k_{\perp}=2,959, \\
\varkappa_{\|}=1,272, & \varkappa_{\perp}=1,212,
\end{array}
$$

Obwohl die Differenzen zwischen den beobachteten und den berechneten Werten von $M$ und $N$ ziemlich groß sind, stimmt das Endresultat mit demjenigen des letzten Versuches sehr gut überein.

3. Gedehnter Stahlspiegel $C$.

Mit dem Spiegel $C$ wurde eine ähnliche Reihe Beobachtungen gemacht. Es wurde beobachtet in allen vier Lagen des Polarisators. Das SchluBergebnis ist in der Tab. XII zusammengebracht.

$$
\text { Tabelle XII. }
$$

Gedehnter Stahl, $\frac{\Delta l}{l}=0,055$.

$\varphi=77^{\circ}$.

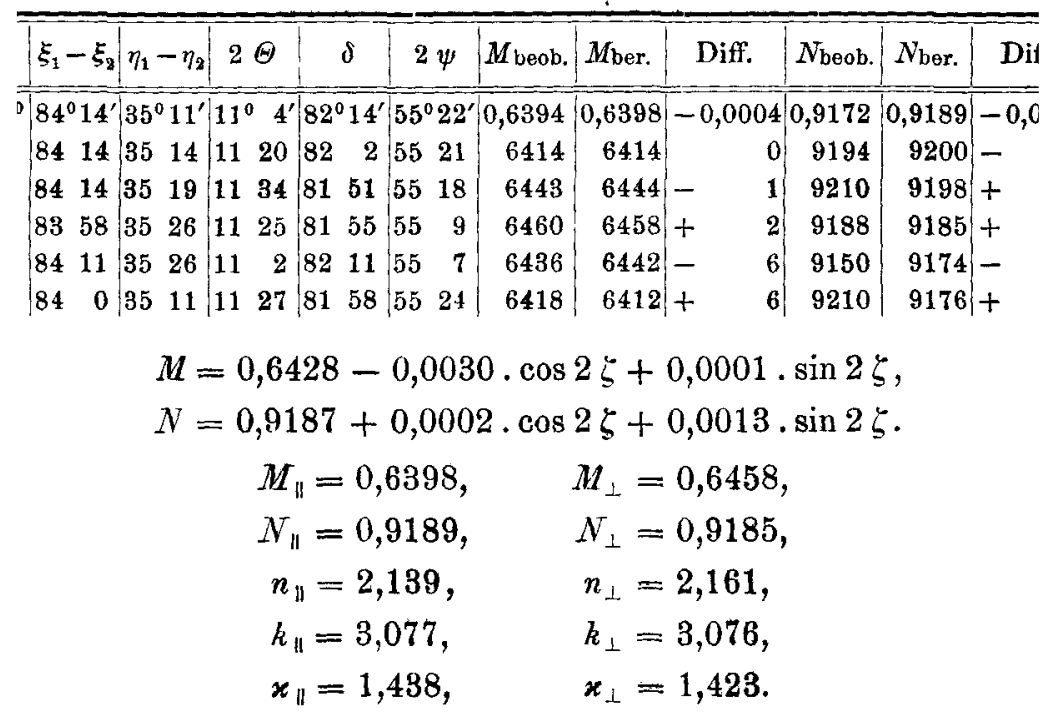


4. Gedehnter Stahl $A$.

Mit dem Spiegel $A$ unter derselben Versuchsanordnung, habe ich das folgende Resultat bekommen.

Tabelle XIII.

Gedehnter Stahl, $\frac{\Delta l}{l}=0,021$.

$\varphi=77^{\circ}$.

\begin{tabular}{|c|c|c|c|c|c|c|c|c|c|c|c|}
\hline$\zeta$ & $\xi_{1}-\xi_{2}$ & $\eta_{1}-\eta_{2}$ & $2 \Theta$ & $\delta$ & $2 \psi$ & $M_{\text {boob }}$ & $M_{\text {ber. }}$ & Diff. & $N_{\text {beob. }}$ & $N_{\text {ber. }}$ & Diff. \\
\hline $0^{0}$ & $84^{\circ} 17^{\prime}$ & $35^{\circ} 44^{\prime}$ & $2^{0} 40^{\prime}$ & $88^{\circ}$ & $54^{\circ} \quad 6$ & 0,6027 & 0,6027 & $+0,0000$ & 0,8320 & 0,8312 & $+0,0008$ \\
\hline 30 & 8426 & $\begin{array}{ll}35 & 47\end{array}$ & 234 & 88 & $54 \quad 4$ & 6028 & 6033 & 5 & 8305 & 8306 & $\begin{array}{ll}- & 1\end{array}$ \\
\hline 60 & $\begin{array}{ll}84 & 9\end{array}$ & $\begin{array}{ll}35 & 56\end{array}$ & 234 & 887 & 53 & 053 & 6033 & 20 & 8295 & 8298 & + \\
\hline 90 & $\begin{array}{ll}84 & 18\end{array}$ & $\begin{array}{lll}35 & 50\end{array}$ & 216 & $\begin{array}{ll}88 & 21\end{array}$ & $53 \quad 59$ & 6020 & 6025 & $1-$ & 8283 & 8286 & - \\
\hline 120 & $84 \quad 19$ & $35 \quad 44$ & 221 & $\begin{array}{ll}88 & 18\end{array}$ & $54 \quad 6$ & 6007 & 6019 & 12 & 8300 & 8292 & $+r$ \\
\hline 150 & $184 \quad 19$ & $\mid \begin{array}{ll}35 & 49\end{array}$ & | 224 & $\begin{array}{ll}88 & 15\end{array}$ & 54 & 6023 & 6019 & + & 8293 & $8305 !$ & 1 \\
\hline
\end{tabular}

$$
\begin{array}{cc}
M=0,6026+0,0001 \cdot \cos 2 \zeta+0,0008 \cdot \sin 2 \zeta, \\
N=0,8299+0,0013 \cdot \cos 2 \zeta+0,0001 \cdot \sin 2 \zeta, \\
M_{\|}=0,6027, & M_{\perp}=0,6025, \\
N_{\|}=0,8312, & N_{\perp}=0,8286, \\
n_{\|}=2,413, & n_{\perp}=2,423, \\
k_{\|}=3,328, & k_{\perp}=3,332, \\
x_{\|}=1,379, & x_{\perp}=1,375 .
\end{array}
$$

\begin{tabular}{|c|c|c|c|c|c|c|c|c|c|c|c|}
\hline$\zeta$ & $\xi_{1}-\xi_{9}$ & $\eta_{1}-\eta_{2}$ & $2 \Theta$ & $\delta$ & $2 \psi$ & $M_{\text {beob }}$ & $M_{\text {ber. }}$ & Diff. & $N_{\text {beab. }}$ & $N_{\text {ber. }}$ & Diff. \\
\hline $0^{0}$ & $84^{0} 50^{\prime}$ & $36^{\circ} 48^{\prime}$ & $1^{\circ} 45^{\prime}$ & $88^{\circ} 41^{\prime}$ & $53^{0} \quad 3^{\prime}$ & 0,6124 & 0,6108 & $+0,0016$ & 0,8135 & 0,8152 & $-0,0017$ \\
\hline 30 & 8436 & $36 \quad 36$ & 26 & $\begin{array}{ll}88 & 26\end{array}$ & $53 \quad 15$ & 6118 & 6120 & - & 8190 & 8165 & 25 \\
\hline 60 & 8451 & $\begin{array}{ll}36 & 44\end{array}$ & 155 & $88 \quad 34$ & 537 & 6124 & 6144 & 20 & 8157 & 8165 & - \\
\hline 90 & 8441 & $\begin{array}{ll}37 & 0\end{array}$ & 25 & $88 \quad 25$ & 5250 & 6177 & 6156 & + & 8145 & 8152 & - \\
\hline 120 & 8449 & $36 \quad 49$ & 156 & 8833 & $53 \quad 2$ & 6137 & 6144 & - & 8148 & 8139 & + \\
\hline 150 & $84 \quad 36$ & $\mid \begin{array}{ll}36 & 42\end{array}$ & 140 & $188 \quad 45$ & 53 & 6105 & 6120 & 15 & 8135 & 8139 & 1- \\
\hline
\end{tabular}

4. Gedehnter Stahlspiegel $B$.

Die Beobachtung mit dem Spiegel $B$ ergab folgendes:

Tabelle XIV.

Gedehnter Stahl, $\frac{\Delta l}{l}=0,108$.

$\varphi=\mathbf{7 7}^{\circ}$. 
Wirkung einer permanenten magnetischen Ausdehnung etc.

$$
\begin{array}{rlrl}
M & =0,6132-0,0024 \cdot \cos 2 \zeta, \\
N & =0,8152+0,0015 \cdot \sin 2 \zeta, \\
M_{\|} & =0,6108, & M_{\perp}=0,6156, \\
N_{\|}=0,8152, & N_{\perp}=0,8152, \\
n_{\|}=2,485, & n_{\perp}=2,490, \\
k_{\|}=3,317, & k_{\perp}=3,298, \\
\boldsymbol{x}_{\|}=1,335, & \varkappa_{\perp}=1,325 .
\end{array}
$$

Frgebnisse.

Das ganze Ergebnis der Versuche ist in der Tab. XV zusammengestellt, wo das Zeichen 0 bedeutet, daß die Größe sich auf den normalen, spannungsfreien Zustand, und die Zeichen

\begin{tabular}{|c|c|c|c|c|c|c|c|c|c|c|c|}
\hline & & $\frac{\Delta l}{l}$ & $n_{0}$ & $n_{\text {if }}$ & $n_{\perp}$ & $k_{0}$ & $k_{\|}$ & $k_{\perp}$ & $x_{0}$ & $x_{1}$ & $x_{\perp}$ \\
\hline $\begin{array}{l}2 \\
3\end{array}$ & $\begin{array}{c}\text { Kupfer } \\
" \\
"\end{array}$ & $\begin{array}{c}0,026 \\
,\end{array}$ & 0,7083 & $\begin{array}{l}0,6982 \\
0,6913\end{array}$ & $\left|\begin{array}{l}0,7073 \\
0,6952\end{array}\right|$ & 1,990 & $\begin{array}{l}1,830 \\
1,824\end{array}$ & $\left|\begin{array}{l}1,817 \\
1,789\end{array}\right|$ & 2,815 & $\begin{array}{l}2,621 \\
2,638\end{array}$ & $\mid \begin{array}{l}2,569 \\
2,574\end{array}$ \\
\hline $\begin{array}{l}4 \\
5\end{array}$ & $\begin{array}{c}\text { Silber } \\
,\end{array}$ & 0,0027 & 0,2934 & 0,2431 & 0,3915 & 3,368 & 3,627 & 3,535 & 11,48 & 14,92 & 9,03 \\
\hline $\begin{array}{l}7 \\
8 \\
9\end{array}$ & $\begin{array}{rc}\text { Stahl } \\
\quad, & D \\
" & D \\
" & A \\
" & C \\
" & B\end{array}$ & $\mid \begin{array}{c}0,013 \\
n \\
0,021 \\
0,055 \\
0,108\end{array}$ & 2,373 & $\begin{array}{l}2,399 \\
2,390 \\
2,413 \\
2,139 \\
2,485\end{array}$ & $\begin{array}{l}2,441 \\
2,424 \\
2,423 \\
2,161 \\
2,490\end{array}$ & 3,337 & $\begin{array}{l}3,052 \\
3,056 \\
3,328 \\
3,077 \\
3,317\end{array}$ & $\mid$\begin{tabular}{|}
2,959 \\
2,953 \\
3,332 \\
3,076 \\
3,298
\end{tabular} & 1,395 & $\begin{array}{l}1,272 \\
1,279 \\
1,379 \\
1,438 \\
1,335\end{array}$ & $\begin{array}{l}1,212 \\
1,219 \\
1,375 \\
1,423 \\
1,325\end{array}$ \\
\hline
\end{tabular}
$\|$ bez. $\perp$, daß sie sich auf den gedehnten Zustand, und zwar auf die Richtung parallel und senkrecht zur mechanischen Spannnung beziehen. $n$ ist der Brechungsexponent, $k$ der Absorptionskoeffizient und $x$ der Absorptionsindex, wobei $k=n \varkappa$ ist.

Tabelle XV.

Man zieht sogleich aus der Tabelle die folgenden Schluß. folgerungen.

1. In allen von mir untersuchten Fällen ist

$$
n_{\|}<n_{\perp} \text {. }
$$


2. Nur einen einzigen Fall von dem Stahlspiegel $A$ ausgenommen, ist

$$
k_{\|}>k_{\perp} \text {. }
$$

Aber da der Unterschied in diesem Falle sehr klein ist, ist es sehr wahrscheinlich, daB die Regel $k_{\|}>k_{\perp}$ allgemeine Gültigkeit besitzt.

3. In allen Fällen ist

$$
x_{\|}>x_{\perp} .
$$

4. Was das Verhältnis zwischen den normalen und gespannten Zuständen betrifft, haben wir

$$
\begin{aligned}
& \text { im Kupfer } n_{\|}<n_{\perp}<n_{0} \text {, } \\
& \text { "Silber } n_{\|}<n_{0}<n_{\perp} \text {, } \\
& \text {, Stahl } n_{0}<n_{\|}<n_{\perp} \text {. }
\end{aligned}
$$

Der Spiegel $C$ bietet die einzige Ausnahme dar, wo die letzte Beziehung nicht erfüllt ist; aber das gesamte Resultat in ihm weicht sehr viel von dem allgemeinen Verlauf $a b$, und es liegt die Vermutung nahe, daB die Oberflächenbeschaffenheit im Spiegel $C$ nicht einwandfrei war. Leider gestattete mir die für meinen Aufenthalt in Göttingen begrenzte Zeit nicht, die Messung zu wiederholen.

5. Für den Absorptionskoeffizient gelten die Beziehungen

während

$$
\text { im Kupfer und Stahl } k_{0}>k_{\|}>k_{\mathrm{T}} \text {; }
$$

$$
\text { im Silber } \quad k_{\|}>k_{\perp}>k_{0},
$$

abgesehen von der kleinen Abweichung im Stahlspiegel $A$.

6. Endlich für den Absorptionsindex sehen wir

$$
\begin{array}{ll}
\text { im Kupfer und Stahl } & x_{0}>x_{\|}>x_{\perp}, \\
\text { " Silber } & x_{\|}>x_{0}>x_{\perp} .
\end{array}
$$

Soviel über die qualitativen Beziehungen. Wenn man die quantitativen Verhältnisse ins Auge faBt, findet man gleichfalls einige interessante Resultate. Hier ist das Verhalten ron Silber sehr auffallend, wie es in bezug auf den Sinn der Änderung singulär war. Obwohl das Probestück am wenigsten 
von allen gedehnt ist, findet man beim Silber doch eine kolossale Änderung der Konstanten, und zwar gilt

$$
\begin{aligned}
& n_{0}: n_{\|}: n_{\perp}=1: 0,83: 1,33, \\
& k_{0}: k_{\|}: k_{\perp}=1: 1,08: 1,05, \\
& x_{0}: x_{\|}: x_{\perp}=1: 1,30: 0,79 .
\end{aligned}
$$

Bei den anderen Metallen sind die Änderungen viel kleiner; sogar im Stahlspiegel $B$, wo das Metall beinahe bis zu seiner Grenze der Ausdehnung gespannt war, haben wir nur

$$
\begin{aligned}
& n_{0}: n_{\|}: n_{\perp}=1: 1,047: 1,049, \\
& k_{0}: k_{\|}: k_{\perp}=1: 0,994: 0,988, \\
& x_{0}: x_{\|}: x_{\perp}=1: 0,957: 0,950 .
\end{aligned}
$$

In bezug auf die numerische Beziehung zwischen den GröBen der mechanischen Elongation und der Änderungen in den optischen Konstanten, kann man leider nicht etwas Bestimmtes feststellen. Die Beobachtungen sind nicht genau genug, oder vielmehr die Herstellung der Spiegel müBte noch sorgfältiger möglich sein.

Am SchluB möchte ich gern noch auf eine Frage aufmerksam machen. Woran liegt eigentlich die beobachtete Anisotropie der optischen Konstanten in gedehnten Metallen? Ist sie von molekularer Ursache, oder von gröberem Charakter? Wir haben oben gesehen, daB man bei einem gewöhnlichen Spiegel durch die matte Politur einen zu großen Brechungsexponenten $n$ und einen zu kleinen Absorptionsindex $x$ erhalten würde. Durch den Zug bekommt ein isotroper Metallspiegel eine faserige Struktur, wie man sie leicht mit einem Mikroskope konstatieren kann. Es liegt die Vermutung nahe, daB durch diese faserige Struktur der Spiegel sich so verhalten wird, als ob seine Politur in der Richtung der Spannung besser wäre wie senkrecht dazu. Daraus folgt ohne weiteres, daB

$$
\begin{aligned}
& n_{\|}<n_{\perp}, \\
& x_{\|}>x_{\perp}
\end{aligned}
$$

sein würde. Ob die beobachtete Anisotropie sich völlig durch diese Annahme erklären läBt, kann man nicht sagen. Vielleicht ist es in Wirklichkeit der Fall, daB die optischen Eigenschaften 
des ausgedehnten Metallspiegels wirklich anisotropisch sind, aber die oben gesagten Umstände auch mitspielen. Es wird sehr lehrreich sein, eine ähnliche Beobachtung auszuführen mit einem sonst isotropischen Metallspiegel, der, z. B. wie mit einem Gitter, mit genau nach einer Richtung laufenden Kratzen bedeckt ist. $\mathrm{DaB}$ durch die Dehnung eine wirkliche optische Anisotropie hervorgerufen worden ist, wird dadurch plausibel, $\mathrm{daB}$ man durch die faserige Struktur allein die Differenz zwischen $n_{0}$ und $n_{\|}$oder $n_{\perp}$ nicht erklären kann. Auch kommen offenbar noch die spezifischen Eigenschaften des betreffenden Metalles in bezug auf die Größe und die Richtung der Änderung in Betracht.

Göttingen, math.-phys. Laboratorium, Mai 1906.

(Eingegangen 15. Juni 1906.) 